\title{
A translational EEG-based approach to assess modulation of long-lasting NMDAR-dependent synaptic plasticity
}

\author{
Jeffrey S. Burgdorf ${ }^{1,2}$ (D) E. P. Christian ${ }^{1} \cdot$ L. Sørensen ${ }^{1} \cdot$ P. K. Stanton ${ }^{3} \cdot$ K. Leaderbrand ${ }^{1,2} \cdot$ T. M. Madsen ${ }^{1}$. \\ M. A. $\operatorname{Khan}^{1} \cdot$ R. A. Kroes ${ }^{1,2}$ - J. R. Moskal ${ }^{1,2}$
}

Received: 29 December 2018 / Accepted: 25 July 2019 / Published online: 7 August 2019

(C) The Author(s) 2019

\begin{abstract}
Background NYX-2925 is a novel N-methyl-D-aspartate receptor (NMDAR) modulator that has been shown to facilitate both NMDAR-dependent long-term potentiation (LTP) in vitro and learning and memory in vivo.

Objective The present studies examine the effects of NYX-2925 on NMDAR-dependent auditory LTP (aLTP) in vivo.

Methods NMDAR-dependent aLTP and NMDAR-dependent auditory mismatch negativity (MMN) was measured, as well as changes in resting-state $\mathrm{qEEG}$ power.

Results NYX-2925 (1, $10 \mathrm{mg} / \mathrm{kg}$ PO) increased aLTP $1 \mathrm{~h}$ after auditory tetanus measured by the post- minus pre-tetanus difference waveform 140-180 ms post tone onset. NYX-2925 (0.1, 1 mg/kg PO) facilitated MMN measured by the difference waveform (i.e., deviant minus standard tones). NYX-2925 (0.1, 1, $10 \mathrm{mg} / \mathrm{kg}$ PO) also enhanced resting-state alpha qEEG power. Conversely, the NMDAR glutamate site antagonist CPP $(10 \mathrm{mg} / \mathrm{kg} \mathrm{IP})$ reduces alpha power and MMN and produces an opposite effect as NYX-2925 on aLTP.

Conclusions Together, these data suggest that the activation of the NMDAR by NYX-2925 enhances synaptic plasticity in vivo, which may both reduce symptoms of neurological disorders and serve as a biomarker for drug effects. This is the first demonstration of a long-lasting (1-h post-tetanus) effect of NMDAR modulation on synaptic plasticity processes in vivo using a noninvasive technique in freely behaving animals.
\end{abstract}

Keywords LTP $\cdot$ Mismatch negativity $\cdot$ EEG $\cdot$ Auditory-evoked potentials $\cdot$ NMDA receptors

\section{Introduction}

NMDA receptor (NMDAR) activity is critical for synaptic plasticity. For example, NMDARs are required for the induction of some forms of long-term potentiation (LTP), a form of a long-lasting increase in synaptic strength that is a putative substrate for learning and memory (Luscher and Malenka 2012). The induction, but not long-term maintenance, of

Jeffrey S. Burgdorf

jeffreyburgdorf@aptinyx.com

1 Aptinyx Inc., 1801 Maple Ave, Suite 4300, Evanston, IL 60201, USA

2 Falk Center for Molecular Therapeutics, Department of Biomedical Engineering, Northwestern University, Evanston, IL 60201, USA

3 Cell Biology \& Anatomy, New York Medical College, Valhalla, NY, USA
LTP at many excitatory synapses in hippocampal and medial prefrontal cortex (MPFC) slices is blocked by the NMDA glutamate receptor antagonist APV, and by NMDAR channel blockers such as MK-801 and ketamine (Bliss and Collingridge 2013; Davis et al. 1992). In addition, NMDAR activation promotes LTP (Zhang et al. 2008). Once LTP has been established, in a process which includes the insertion of AMPA receptors into synapses in a protein synthesisdependent manner, the increased synaptic effect that is the expression of LTP is not blocked by NMDAR antagonists (Luscher and Malenka 2012).

In vivo, learning acquisition and long-term memory formation of hippocampus- and MPFC-dependent tasks are also blocked by NMDAR antagonists (Burgdorf et al. 2011; Davis et al. 1992). In vivo electrical stimulus-induced LTP is also NMDAR-dependent, can be blocked by the NMDAR antagonist APV (Morris 1989), and is facilitated by application of the NMDAR agonist D-cycloserine (DCS; (Hopkins et al. 2013)). 
NMDAR activation is essential for a large portion of LTP induced by tetanization with sound or visual stimuli, as recorded by sensory-evoked potentials via scalp EEG electrodes (Sanders et al. 2018). NMDAR activation with DCS facilitates short-term potentiation (Forsyth et al. 2015), and inactivation with CPP (an analog of APV) inhibits the induction of LTP (Clapp et al. 2006). Here, in order to determine whether NMDAR modulation can facilitate LTP in vivo, a modulator of the NMDAR with long-lasting properties was used.

NYX-2925 ((2S,3R)-3-hydroxy-2-((R)-5-isobutyryl-1oxo-2,5-diazaspiro[3.4]octan-2-yl)butanamide) is a novel NMDAR modulator (Khan et al. 2018; Moskal et al. 2017). Supported by robust preclinical data in animal models of neuropathic pain (Ghoreishi-Haack et al. 2018), NYX-2925 is currently being investigated in two phase II clinical studies for the treatment of painful diabetic peripheral neuropathy and fibromyalgia. Previous studies demonstrated that NYX-2925 is a member of a novel class of NMDAR-specific modulators that facilitate NMDAR-dependent processes associated with learning and memory (Khan et al. 2018). Ex vivo, NYX-2925 enhances hippocampal and MPFC LTP and inhibits the induction of long-term depression (LTD) at concentrations that facilitate NMDAR current (Khan et al. 2018). Persistent changes in long-lasting plasticity are also seen $24 \mathrm{~h}$ post dosing in vivo, as measured by facilitation of ex vivo hippocampal LTP and mature dendritic spine morphology that far outlasts drug presence (Khan et al. 2018). In vivo, NYX-2925 enhances NMDAR-dependent positive emotional learning and this effect is blocked by the NMDAR glutamate site antagonist CPP. Oral doses of NYX-2925 in vivo that increase learning and memory produce CSF concentrations that enhance NMDAR current in vitro (Khan et al. 2018).

Therefore, an in vivo translational EEG approach was used to evaluate the long-lasting effects of NYX-2925induced NMDAR modulation on synaptic plasticity changes. This approach should be directly applicable to similar studies conducted in human subjects and provide new insights into NMDAR-mediated synaptic plasticity mechanisms and their role in CNS disorders (Clapp et al. 2012; Clapp et al. 2005), such as centrally mediated chronic pain (e.g., fibromyalgia), post-traumatic stress disorder, and cognitive impairment.

The experiments in this study were designed to test whether NYX-2925 could enhance auditory-evoked responses $1 \mathrm{~h}$ after auditory tetanus (aLTP). As part of this experiment, NMDAR-dependent mismatch negativity (MMN), the auditory event-related potential to a deviant tone compared with a standard tone, was also examined, as well as resting quantitative EEG (qEEG) power across the alpha frequency band.

\section{Materials and methods}

\section{Animals}

Male 2-3-month-old Sprague-Dawley (SD) rats from Envigo (USA) were used. Rats were housed in Lucite cages with aspen wood chip bedding, maintained on a 12:12 light:dark cycle (lights on at $6 \mathrm{am}$ ) and given ad libitum access to Teklad lab chow (Envigo, USA) and tap water throughout the study. All experiments were approved by the Northwestern University Animal Care and Use Committee.

\section{Surgeries, data acquisition, and analysis}

Rats were anesthetized with isoflurane (5\% induction and 2-3\% maintenance; $15-20 \mathrm{~min}$ total duration) and implanted with skull screws to record cortical EEG (Pinnacle, USA). Animals were given 7 days to recover before the start of testing. Auditory event-related potentials (ERPs) were recorded from a frontal cortex skull screw using a cerebellar skull screw as a ground/reference. EEG signals were captured via a tethered system (Pinnacle, USA). Data were acquired at $1 \mathrm{kHz}$ using an A\&M (USA) amplifier with a high $(0.1 \mathrm{~Hz})$ - and low-pass $(100 \mathrm{~Hz})$ filters and digitized using Data Wave (USA) acquisition software. Data were analyzed using Brain Products Analyzer 2 software (Germany).

Animals were dosed with NYX-2925 $(0.1,1,10 \mathrm{mg} / \mathrm{kg}$ PO; Sai Life Sciences, India) or $0.5 \%$ carboxymethylcellulose (Sigma, USA) in distilled deionized water (Millipore, USA). NYX-2925 was administered orally so that these preclinical data can be more directly compared with a parallel human study with NYX-2925 that also used oral dosing. An additional group of rats received the NMDAR glutamate site antagonist CPP (10 mg/kg IP; Sigma USA) or distilled deionized water vehicle. This dose of CPP was shown to block the learning and memory effects of NYX-2925 (Khan et al. 2018) as well as block in vivo hippocampal LTP (Abraham and Mason 1988).

\section{Quantitative EEG acquisition}

Within $5 \mathrm{~min}$ of dosing, rats were tethered and placed into individual sound-attenuated chambers under dim illumination. For the first 60-min post-dosing, no external stimulation was given (resting state). qEEG data from the first 60-min post-dosing was analyzed across the entire period using a Hanning window $(2 \mathrm{~s}, 0.5-\mathrm{Hz}$ resolution). Relative (1.5 to $40 \mathrm{~Hz})$ voltage spectral density $(\mu \mathrm{V} / \mathrm{Hz})$ was used for the analysis. 


\section{MMN paradigm}

Rats were placed in cylindrical test cages $(30.5 \mathrm{~cm}$ diameter, $30.5 \mathrm{~cm}$ high clear Acrylic cylinder; Pinnacle USA). Testing consisted of standard $(6 \mathrm{kHz})$ or deviant $(8 \mathrm{kHz})$ tone pips $(50-$ $\mathrm{ms}$ duration) and an interstimulus interval of $350 \mathrm{~ms}$, presented in a pseudorandom manner, with at least 2 standards occurring in a row. Tones were presented at $85 \mathrm{~dB}$ using a speaker (Avisoft, Germany) placed on the floor of the cage. One trial consisted of 1800 standard tones and 200 deviant tones (total, 2000 tones). Averaged standard and deviant waveforms were baselined to the pre-stimulus period $(-50$ to $0 \mathrm{~ms}$ before tone onset). A difference wave was obtained by subtracting the standard from the deviant waveform and was used as the measure of MMN. The range used for calculating MMN was determined based on the pre-dose MMN difference grand average waveform for all of the animals tested (50-250 ms) as shown in Fig. 1B. MMN was calculated as the average microvolt value from 50 to $250 \mathrm{~ms}$ after the tone onset from the deviant-standard difference wave.

\section{aLTP induction stimulus paradigm}

aLTP was induced by a high-frequency train $(3 \times 10 \mathrm{~Hz}$ for $5 \mathrm{~min} ; 3000$ tones) of auditory stimuli ( $6 \mathrm{kHz}, 50-\mathrm{ms}$ duration, $85 \mathrm{~dB}$ ), using a paradigm similar to the one described by Clapp and colleagues (Clapp et al. 2006). MMN testing occurred immediately before tetanus (pre-tetanus) and $1 \mathrm{~h}$ after tetanus (post-tetanus). Post- minus pre-tetanus difference waves were generated to determine the range (in milliseconds) in which aLTP occurred for both the standard and deviant tone as defined by the largest mean difference and statistical difference (within-subject $t$ test) in the vehicle group pre- vs. posttetanus. Using this criterion, aLTP occurred within the range a
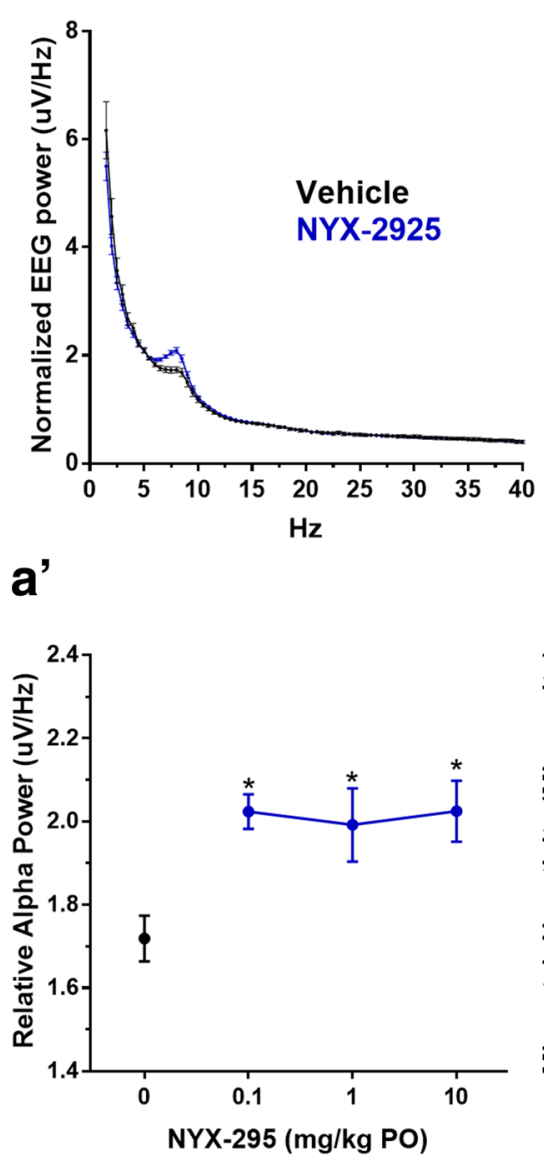

b

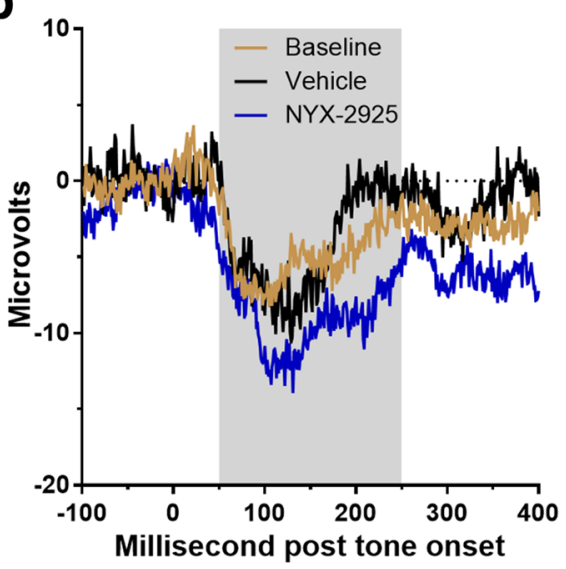

b'

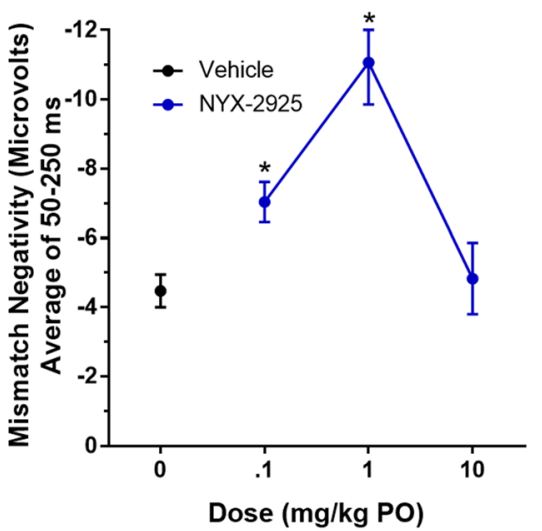

C
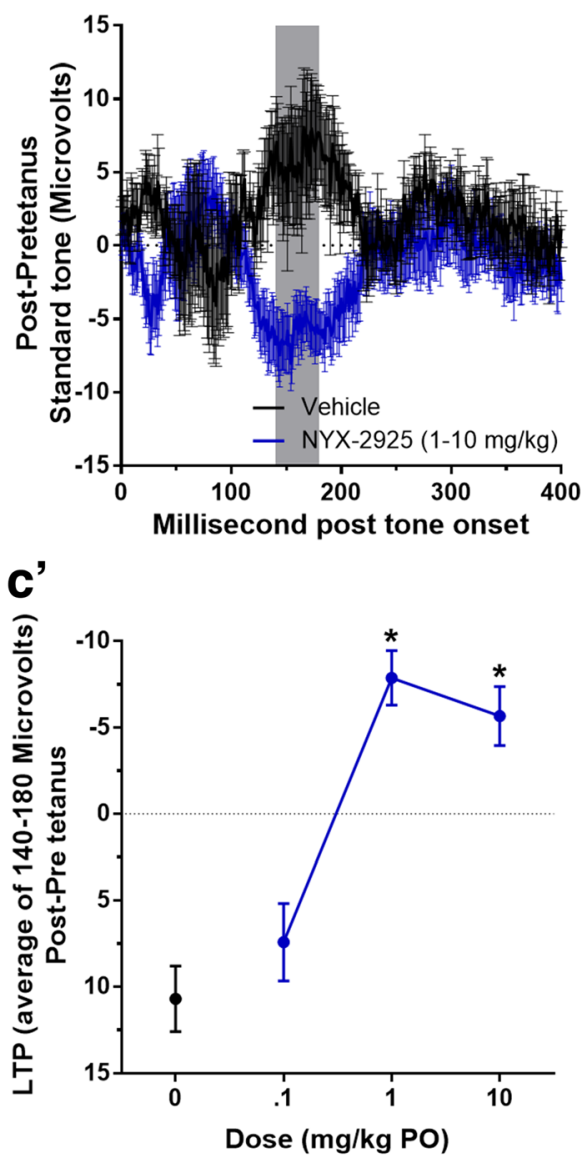

Fig. 1 NYX-2925 enhances resting alpha power (qEEG), mismatch negativity (MMN), and auditory-induced long-term potentiation (aLTP). (A, A') Average power spectral density plots showing that NYX-2925 $(0.1,1,10 \mathrm{mg} / \mathrm{kg}$ PO) enhanced alpha power $(7-8.5 \mathrm{~Hz})$ compared with vehicle without affecting power in the other EEG bands (data not shown). (B, B') Grand average MMN waveforms for showing that NYX-2925 $(0.1,1 \mathrm{mg} / \mathrm{kg} \mathrm{PO})$ enhanced MMN compared with vehicle from 50 to $250 \mathrm{~ms}$ after tone onset. (C, C') Average post-pre tetanus waveforms show that NYX-2925 (1, 10 mg/kg PO) enhanced aLTP 140-180 ms after tone onset. This range was used given that this was the region in which the vehicle group showed the largest post-pre tetanus change. (B) LTP was enhanced in the 1 and $10 \mathrm{mg}$ dose groups $(p<.05)$. Using a similar methodology (Clapp et al. 2006), an NMDAR antagonist has been shown to inhibit sensory-induced LTP. Mean \pm SEM. $* p<.05$ Bonferroni post hoc test vs. vehicle following a one-way ANOVA. $n=7-10$ per group 
of 140-180 ms after the tone onset in the vehicle group based on the average of the standard and deviant tone waveforms, which is consistent with the range (ms) and generalization (both standard and deviant tone) seen in humans with aLTP using a MMN paradigm (Kompus and Westerhausen 2018). The average microvolt value across $140-180 \mathrm{~ms}$ for both the standard and deviant tone was used to calculate effects of aLTP on both the NYX-2925 and vehicle groups and determine possible drug effects.

\section{Experimental timeline}

On the day before testing, animals received a habituation session of qEEG and MMN. These data were used to randomize animals into treatment groups. On the testing day, $10 \mathrm{~min}$ of qEEG was first collected followed by MMN. Animals were then dosed with NYX-2925 (0, 0.1, 1, $10 \mathrm{mg} / \mathrm{kg}$ PO) or the NMDAR glutamate site antagonist CPP $(0,10 \mathrm{mg} / \mathrm{kg}$ IP). Immediately after dosing, $1 \mathrm{~h}$ of qEEG was collected and used for the qEEG measures shown in Figs. 1A, $\mathrm{A}^{\prime}$ and 2A. Immediately after qEEG testing, rats received $\mathrm{MMN}$ testing, and these data are shown in Figs. 1B, B' and 2B. Animals then received 3 auditory tetani (each consisting of a 5-min tetanus with a 10-min inter-tetani interval). One hour after the first tetanus, the MMN paradigm was administered again and post-pre tetanus auditory-evoked responses were used for calculating aLTP (data shown in Figs. 1C, C' and 2C).

\section{Statistical analysis}

The effect of NMDAR activation on qEEG and MMN variables was analyzed by a separate one-way analysis of variance (ANOVA) test with a null hypothesis of no difference in the dependent variable, with respect to the independent variable of each of the 4 doses (NYX-2925
0.1 , 1 , and $10 \mathrm{mg} / \mathrm{kg}$ PO, and vehicle). For the aLTP study, a within-subject component was also used (postpre tetanus difference scores were calculated). All statistical tests were two-sided with significance set at $p<.05$. For any ANOVA test indicating significance, pairwise comparisons using $t$ tests were applied between doses. These pairwise comparisons were adjusted for multiplicity using the Bonferroni correction.

Likewise, CPPs were analyzed against vehicle using the one-way ANOVA. All analyses were conducted with Statview (USA) software. Individual datapoints from unstable EEG recordings were excluded from the analysis.

\section{Results}

qEEG measures revealed a significant modulation of the alpha range $(7-8.5 \mathrm{~Hz})(F(3,31)=5.72 p<.01)$ in response to acute exposure with NYX-2925. All NYX-2925 dose levels showed a significantly increased mean alpha voltage density compared with vehicle, confirmed by the Bonferroni adjusted multiple comparisons (NYX-2925 0.1, 1, 10 mg/kg PO vs. vehicle $p<.05$ ), shown in Fig. 1A'.

Grand average voltage spectral density plots for NYX$2925(0.1,1,10 \mathrm{mg} / \mathrm{kg})$ and vehicle are shown in Fig. 1A.

Average MMN amplitude (50-250 ms) was significantly modulated between groups $(F(3,25)=11.59 p<.001)$ in response to acute exposure of NYX-2925. NYX-2925 (1 mg/kg PO) showed a significantly enhanced MMN amplitude compared with vehicle, confirmed by the Bonferroni adjusted multiple comparisons (NYX-2925 $1 \mathrm{mg} / \mathrm{kg}$ PO vs. vehicle $p<.001)$, shown in Fig. 1B'.

Average waveforms for the deviant-standard difference wave (MMN) for NYX-2925 $(0.1,1 \mathrm{mg} / \mathrm{kg})$ and vehicle are shown in Fig. 1B.
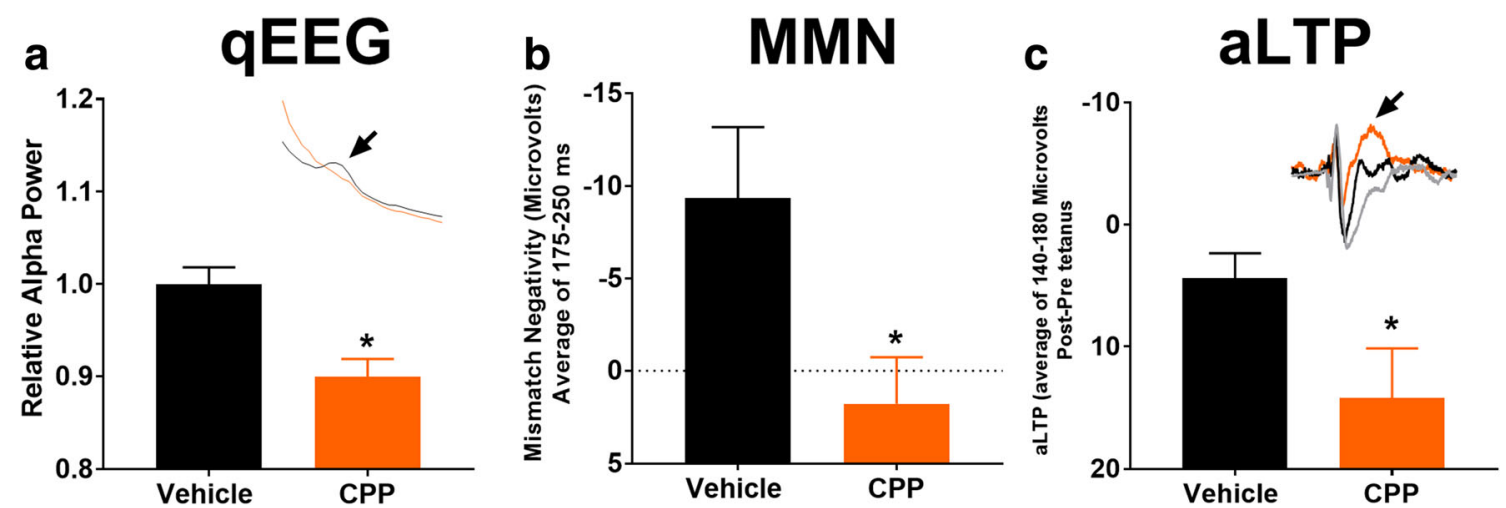

Fig. 2 The NMDAR glutamate site antagonist CPP inhibited resting alpha power, MMN, and aLTP. CPP inhibited resting alpha power (A), MMN (B), and increased aLTP-induced positivity (C). NYX-2925 (Fig. 1) had the opposite effect on all three of these measures. Insets: (A) alpha peak (arrow), showing the CPP nearly eliminates the alpha peak; (C) effect of tetanus on aLTP from the grand average of the standard and deviant waveforms with gray being the pre-tetanus waveform. CPP produces a near maximal positive deflection across the aLTP window (140$180 \mathrm{~ms}$; arrow). ${ }^{*} p<.05$ one-way ANOVA, CPP vs. vehicle. $n=9-10$ per group 
The aLTP protocol, inducing auditory synaptic plasticity, modulated the standard tone waveform, measured by the mean amplitude from 140 to $180 \mathrm{~ms}(F(3,32)=24.62$ $p<.001)$. NYX-2925 (1, $10 \mathrm{mg} / \mathrm{kg}$ PO) significantly increased the mean negativity in this range compared with vehicle, confirmed by the Bonferroni adjusted multiple comparisons (NYX-2925 1, $10 \mathrm{mg} / \mathrm{kg}$ PO vs. vehicle $p<.001$ ), shown in Fig. 1C'.

Average post- minus pre-aLTP waveforms for the active doses $(0.1$ and $1 \mathrm{mg} / \mathrm{kg})$ and vehicle are shown in Fig. 1C.

The NMDAR glutamate site antagonist, CPP, produced the opposite effects on each of the EEG variables (Fig. 2) used to evaluate positive modulation of the NMDAR with NYX-2925. CPP inhibited resting alpha power from 7 to $8.5 \mathrm{~Hz}(F(1,18)=$ $14.05 p<.01$; Fig. 2A), whereas NYX-2925 enhanced alpha power (Fig. 1A). CPP also inhibited the latter segment of the MMN waveform (175-250 ms post tone onset) $(F(1,18)=5.89$ $p<.05$; Fig. 2B), which NYX-2925 preferentially enhanced (Fig. 1B). CPP also increased aLTP-induced positivity (140$180 \mathrm{~ms}$ post tone onset) $(F(1,16)=4.68, p<.05$; Fig. $2 \mathrm{C})$, whereas NYX-2925 produced negativity across this same range (Fig. 1C).

EEG response during the auditory tetanus stimulation was also examined. The EEG was visualized by transforming data and recorded during auditory tetanus, to the time-frequency domain using the Morlet wavelets (Morlet parameter $=3$, optimized for the best time sensitivity). This inspection showed that a $6-7-\mathrm{Hz}$ band best followed the auditory stimulation in that a power in the band was seen in a burst that occurred approximately 10 per second and were evenly spaced out, resembling the $10-\mathrm{Hz}$ tone presentation (50-ms tone duration). These 6-7-Hz bursts occurred primarily within the first $160 \mathrm{~s}$ of the 5-min tetanus. Thus, a Fourier analysis of the first $160 \mathrm{~s}$ of the tetanus was employed and relative power was measured from 6 to $7 \mathrm{~Hz}$. The 6-7-Hz band was significantly modulated between groups $(F(2,54)=19.3, p<.05)$, with NYX-2925 $(0.1,1,10 \mathrm{mg} / \mathrm{kg}$ PO) showing a significant enhancement $(p<.05)$ and CPP showing a significant decrease $(p<.05)$ compared with vehicle, suggested by the Bonferroni adjusted multiple comparisons.

\section{Discussion}

This is the first demonstration that positive modulation of the NMDAR facilitates NMDAR-dependent sensory-evoked LTP in vivo. Across auditory and visual modalities, NMDARdependent LTP has typically been defined as persisting for at least $1 \mathrm{~h}$ after sensory tetanus. Potentiation of this duration requires NMDAR activation, is blocked by NMDAR antagonists, is AMPAR-dependent, and requires protein synthesis, whereas short-term potentiation does not typically require NMDAR activation and involves only transient activation of
AMPAR, not insertion of new AMPARs into the postsynaptic membrane (Malenka and Bear 2004). Given that NYX-2925 enhances NMDAR activity, which leads to long-lasting synaptic plasticity, learning acquisition, and long-term memory formation (Khan et al. 2018), it is an ideal molecule for testing in an in vivo NMDAR-dependent LTP assay.

The ability of NYX-2925 to enhance NMDAR-dependent plasticity in vivo was independently confirmed by the MMN paradigm and qEEG studies in response to the aLTP protocol. NMDAR-dependent MMN was facilitated by NYX-2925 both acutely and $1 \mathrm{~h}$ after aLTP. Using this paradigm, NMDAR antagonists have been shown to reduce (Ehrlichman et al. 2008; Sivarao et al. 2014), and glycine (which, among other actions, is a co-agonist at the NMDAR) to facilitate, MMN (Greenwood et al. 2018). The present studies with NYX-2925 further validate the NMDAR dependence of MMN.

The alpha band has been defined as the primary resting-state EEG rhythm, and using this definition, alpha power is positively correlated with increased attention (Klimesch 1999). In addition, enhancing alpha power with repetitive transcranial magnetic stimulation (Klimesch et al. 2003; Romei et al. 2010) or neurofeedback training (Hanslmayr et al. 2005; Zoefel et al. 2011) can improve cognitive task performance. Thus, resting alpha synchronization has predictive power for subsequent sensory experience or cognitive processing, whereas attention to sensory stimuli desynchronizes evoked alpha rhythm and this desynchronization is greater with increasing attention to stimuli (Engel et al. 2001; Silvanto et al. 2008). NYX-2925 enhanced resting alpha power $(7-8.5 \mathrm{~Hz})$. As alpha power may reflect an attentional vigilance state that primes an animal for sensory processing (e.g., MMN and aLTP), increased resting/spontaneous alpha power in the rat EEG may represent a mechanism by which NYX-2925 enhances learning and memory through facilitating attention.

Resting alpha power, MMN, and aLTP are NMDARdependent phenomena given that they are inhibited by the NMDAR glutamate site antagonist CPP. The NMDARdependent variables showed a dynamic activation, seen as the inverted U-shaped dose-response relationship (Fig. 1B', C'), suggesting that various brain circuits and mechanisms are affected at different dose levels of NYX-2925 exposure. They may be modulated by different NMDAR receptor subtypes given that NYX2925 shows a different dose-response across each of these assays, and NYX-2925 enhances NMDAR subtype activity with different potencies (NR2B $>\mathrm{NR} 2 \mathrm{~A}>\mathrm{NR} 2 \mathrm{C}=\mathrm{NR} 2 \mathrm{D})$ as reported in Khan et al. (2018). Therefore, it is possible that alpha power and MMN may be more NR2B-mediated than aLTP. Interestingly, the late temporal range for MMN (175-250 ms post tone onset) that is most sensitive to habituation across testing days (Fig. 1B) is also the most sensitive to NMDAR activation (Figs. 1B and 2B). The NMDAR dependence of aLTP also is seen in response to the tetanus, with positive NMDAR modulation facilitating the entrainment of the EEG response to stimulus 
and NMDAR antagonisms inhibiting this response. Lastly, MMN and aLTP occur across a different time window with MMN being later (175-250 ms) than aLTP (140-180 ms), suggesting that these processes may underlie different neuronal populations.

NYX-2925 is a member of a novel platform of NMDAR modulators, four of which are now in human clinical studies. This is the first study demonstrating that NYX-2925 acts as an NMDAR modulator in vivo and establishes a method for measuring the long-lasting effects of NMDAR activation using a method readily translatable to noninvasive studies in humans. Such studies will provide new insights into NMDAR-mediated synaptic plasticity mechanisms and their role in CNS disorders such as centrally mediated chronic pain (e.g., fibromyalgia), PTSD, and cognitive impairment.

Funding information J.R. Moskal was supported by NIH grant NS100173. J.S. Burgdorf was supported by NIH grants MH094835 and CA199928.

\section{Compliance with ethical standards}

All experiments were approved by the Northwestern University Animal Care and Use Committee.

Conflict of interest The authors declare that they have no conflict of interest.

Open Access This article is distributed under the terms of the Creative Commons Attribution 4.0 International License (http:// creativecommons.org/licenses/by/4.0/), which permits unrestricted use, distribution, and reproduction in any medium, provided you give appropriate credit to the original author(s) and the source, provide a link to the Creative Commons license, and indicate if changes were made.

\section{References}

Abraham WC, Mason SE (1988) Effects of the NMDA receptor/channel antagonists CPP and MK801 on hippocampal field potentials and long-term potentiation in anesthetized rats. Brain Res 462:40-46

Bliss TV, Collingridge GL (2013) Expression of NMDA receptordependent LTP in the hippocampus: bridging the divide. Mol Brain 6:5

Burgdorf J, Kroes RA, Weiss C, Oh MM, Disterhoft JF, Brudzynski SM, Panksepp J, Moskal JR (2011) Positive emotional learning is regulated in the medial prefrontal cortex by GluN2B-containing NMDA receptors. Neuroscience 192:515-523

Clapp WC, Kirk IJ, Hamm JP, Shepherd D, Teyler TJ (2005) Induction of LTP in the human auditory cortex by sensory stimulation. Eur J Neurosci 22:1135-1140

Clapp WC, Eckert MJ, Teyler TJ, Abraham WC (2006) Rapid visual stimulation induces N-methyl-D-aspartate receptor-dependent sensory long-term potentiation in the rat cortex. Neuroreport 17:511515

Clapp WC, Hamm JP, Kirk IJ, Teyler TJ (2012) Translating long-term potentiation from animals to humans: a novel method for noninvasive assessment of cortical plasticity. Biol Psychiatry 71:496-502
Davis S, Butcher S, Morris R (1992) The NMDA receptor antagonist D2-amino-5-phosphonopentanoate (D-AP5) impairs spatial learning and LTP in vivo at intracerebral concentrations comparable to those that block LTP in vitro. J Neurosci 12:21-34

Ehrlichman RS, Maxwell CR, Majumdar S, Siegel SJ (2008) Devianceelicited changes in event-related potentials are attenuated by ketamine in mice. J Cogn Neurosci 20:1403-1414

Engel AK, Fries P, Singer W (2001) Dynamic predictions: oscillations and synchrony in top-down processing. Nat Rev Neurosci 2:704 716

Forsyth JK, Bachman P, Mathalon DH, Roach BJ, Asarnow RF (2015) Augmenting NMDA receptor signaling boosts experiencedependent neuroplasticity in the adult human brain. Proc Natl Acad Sci U S A 112:15331-15336

Ghoreishi-Haack N, Priebe JM, Aguado JD, Colechio EM, Burgdorf JS, Bowers MS, Cearley CN, Khan MA, Moskal JR (2018) NYX-2925 is a novel N-methyl-d-aspartate receptor modulator that induces rapid and long-lasting analgesia in rat models of neuropathic pain. $\mathrm{J}$ Pharmacol Exp Ther 366:485-497

Greenwood LM, Leung S, Michie PT, Green A, Nathan PJ, Fitzgerald P, Johnston P, Solowij N, Kulkarni J, Croft RJ (2018) The effects of glycine on auditory mismatch negativity in schizophrenia. Schizophr Res 191:61-69

Hanslmayr S, Sauseng P, Doppelmayr M, Schabus M, Klimesch W (2005) Increasing individual upper alpha power by neurofeedback improves cognitive performance in human subjects. Appl Psychophysiol Biofeedback 30:1-10

Hopkins SC, Campbell UC, Heffernan ML, Spear KL, Jeggo RD, Spanswick DC, Varney MA, Large TH (2013) Effects of D-amino acid oxidase inhibition on memory performance and long-term potentiation in vivo. Pharmacol Res Perspect 1:e00007

Khan MA, Houck DR, Gross AL, Zhang XL, Cearley C, Madsen TM, Kroes RA, Stanton PK, Burgdorf J, Moskal JR (2018) NYX-2925 is a novel NMDA receptor-specific spirocyclic-beta-lactam that modulates synaptic plasticity processes associated with learning and memory. Int J Neuropsychopharmacol 21:242-254

Klimesch W (1999) EEG alpha and theta oscillations reflect cognitive and memory performance: a review and analysis. Brain Res Brain Res Rev 29:169-195

Klimesch W, Sauseng P, Gerloff C (2003) Enhancing cognitive performance with repetitive transcranial magnetic stimulation at human individual alpha frequency. Eur J Neurosci 17:1129-1133

Kompus K, Westerhausen R (2018) Increased MMN amplitude following passive perceptual learning with LTP-like rapid stimulation. Neurosci Lett 666:28-31

Luscher C, Malenka RC (2012) NMDA receptor-dependent long-term potentiation and long-term depression (LTP/LTD). Cold Spring Harb Perspect Biol 4:a005710

Malenka RC, Bear MF (2004) LTP and LTD: an embarrassment of riches. Neuron 44:5-21

Morris RG (1989) Synaptic plasticity and learning: selective impairment of learning rats and blockade of long-term potentiation in vivo by the N-methyl-D-aspartate receptor antagonist AP5. J Neurosci 9:3040 3057

Moskal JR, Burgdorf JS, Stanton PK, Kroes RA, Disterhoft JF, Burch RM, Khan MA (2017) The development of rapastinel (formerly GLYX-13); a rapid acting and long lasting antidepressant. Curr Neuropharmacol 15:47-56

Romei V, Gross J, Thut G (2010) On the role of prestimulus alpha rhythms over occipito-parietal areas in visual input regulation: correlation or causation? J Neurosci 30:8692-8697

Sanders PJ, Thompson B, Corballis PM, Maslin M, Searchfield GD (2018) A review of plasticity induced by auditory and visual tetanicstimulation in humans. Eur J Neurosci 48(4):2084-2097. https://doi.org/10.1111/ejn.14080 
Silvanto J, Muggleton N, Walsh V (2008) State-dependency in brain stimulation studies of perception and cognition. Trends Cogn Sci $12: 447-454$

Sivarao DV, Chen P, Yang Y, Li YW, Pieschl R, Ahlijanian MK (2014) NR2B antagonist CP-101,606 abolishes pitch-mediated deviance detection in awake rats. Front Psych 5:96

Zhang XL, Sullivan JA, Moskal JR, Stanton PK (2008) A NMDA receptor glycine site partial agonist, GLYX-13, simultaneously enhances
LTP and reduces LTD at Schaffer collateral-CA1 synapses in hippocampus. Neuropharmacology 55:1238-1250

Zoefel B, Huster RJ, Herrmann CS (2011) Neurofeedback training of the upper alpha frequency band in EEG improves cognitive performance. Neuroimage 54:1427-1431

Publisher's note Springer Nature remains neutral with regard to jurisdictional claims in published maps and institutional affiliations. 